\title{
Sensitivity Analysis and Optimization of a Standing Wave Ultrasonic Linear Motor
}

\author{
José M. Fernandez and Yves Perriard
}

\begin{abstract}
This paper presents the sensitivity analysis of an ultrasonic linear motor using design of experiments (DOE) and the finite element (FE) optimization of its deformation amplitude. A first ultrasonic linear motor prototype has been built at the laboratory. A deformation amplitude of about $6.6 \mu \mathrm{m}$ can be obtained by applying a $100 \mathrm{~V}$ voltage. The goal is to obtain a bigger deformation amplitude by varying the motor parameters, in particular the vibratory piece dimensions. First of all, a parametrization of the motor structure is carried out. Then, with the aim of reducing the variation ranges of the input parameters-but also to avoid performing a large number of simulations-a preoptimization stage is necessary. Thus, sensitivity analysis is carried out using design of experiments, which is a good way to obtain the influence of the input parameters on the objective function. Factorial designs have been chosen to find out the effects of each input factor but also the effect of their interactions. This method then is compared with Doehlert design technique, which is generally used for optimization approaches. The results show that it is absolutely necessary to take into account the quadratic terms in the model because they represent an important effect. The use of design of experiments revealed to be an interesting way to analyze numerically the ultrasonic motor as a preoptimization stage and already allows one to improve the deformation amplitude but also to reduce the input parameter variation ranges. Different FE optimization methods are then applied, and results show that the deformation amplitude can be increased by a factor higher than 10 compared to the initial design.
\end{abstract}

\section{INTRODUCTION}

$\mathrm{U}$ LTRASONIC motors are a good alternative to conventional electromagnetic motors because they provide a large output torque but also a braking force without energy consumption [1], [2]. The standing-wave type ultrasonic motor presented in this paper is composed of a vibratory piece that is connected to two piezoelectric drivers and which tip portion generates flat-elliptical movement. A slider, which is pressed against the vibrating body by a prestressing force, can move linearly thanks to the friction forces present at the interface between the stator and the slider. A first ultrasonic linear motor prototype has been built at the laboratory. A deformation amplitude of about $6 \mu \mathrm{m}$ can be obtained by applying a $100 \mathrm{~V}$ voltage to the piezoceramics. The goal is to obtain a bigger deformation amplitude by varying the motor parameters, in particular

Manuscript received October 20, 2005; accepted February 2, 2006. The authors are at the Ecole Polytechnique Fédérale de Lausanne (EPFL) Institut de Production et Robotique (IPR), Laboratoire d'Actionneurs Intégrés (LAI) CH-1015 Lausanne, Switzerland (email: jose.fernandezlopez@epfl.ch) the vibratory piece dimensions. First of all, a parametrization of the motor structure is carried out. Then, with the aim of reducing the variation ranges of the input parameters but also to avoid performing a large number of simulations, a preoptimization stage is necessary. Thus, sensitivity analysis is carried out using design of experiments, which is a good way to obtain the influence of the input parameters on the objective function (deformation amplitude). Sensitivity analysis refers to any numerical strategy that aims to get insight of the influence of input factors on the simulation output. Recently, it is becoming a research subject in itself, and new methods are emerging to answer an urging demand originated in the every day, more larger use of computer modeling [3].

Two different techniques have been used in this study. The first one, using factorial designs, consists of an identification method for additive models with interactions (combination between two or more input parameters) while varying them simultaneously. The second one (Doehlert design) will allow one to know the behavior of our system on an entire experimental field by carrying out many simulations distributed in the field in question. In this manner, it will be possible to predict the result in a point in which no simulation was made (by interpolation) using response surfaces. These techniques have been integrated in Matlab (MathWorks, Inc., Natick, MA) and can interface with the finite element (FE) simulation program Ansys (ANSYS, Inc., Canonsburg, PA) working in batch mode. The optimization study, based on the results obtained thanks to the preoptimization stage, is realized using the finite element method (FEM) Ansys software. The structure obtained then is analyzed to validate the optimization method.

\section{Structure And Modeling of the Ultrasonic LINEAR MOTOR}

\section{A. Introduction}

Modeling of the motor has been performed using FEM analysis, which is one of the most effective numerical methods for engineering problem solving. The aim of numerical modeling is to calculate natural frequencies and modal shapes of the motor and to perform harmonic or transient response analysis. Basic equations for the motion of the motor can be written in matrix form as follows:

$$
\begin{array}{r}
{[M] \frac{\partial^{2}\{u\}}{\partial t^{2}}+[C] \frac{\partial\{u\}}{\partial t}+\left[K_{1}\right]\{u\}+\left[K_{2}\right]\{\Phi\}=\{F\}} \\
{\left[K_{2}\right]^{T}\{u\}+\left[K_{3}\right]\{\Phi\}=\{Q\}}
\end{array}
$$




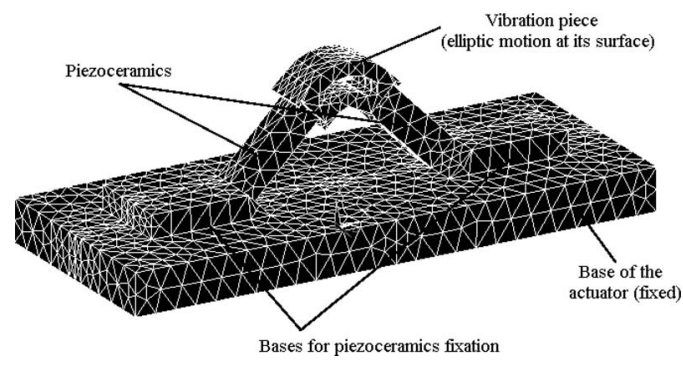

Fig. 1. Ultrasonic linear motor.

TABLE I

Dimensions and Materials of the Simulated Motor.

\begin{tabular}{cc}
\hline Parameter & Value \\
\hline $\mathrm{D}$ & $4.4 \mathrm{~mm}$ \\
$\mathrm{~B}$ & $3 \mathrm{~mm}$ \\
$\mathrm{H}$ & $2 \mathrm{~mm}$ \\
$\mathrm{~L}$ & $9 \mathrm{~mm}$ \\
$\mathrm{E}$ & $1.55 \mathrm{~mm}$ \\
$\mathrm{G}$ & $0.5 \mathrm{~mm}$ \\
$\alpha$ & $45^{\circ}$ \\
$\mathrm{C}$ & $1.4 \mathrm{~mm}$ \\
$R_{1}$ & $1.2 \mathrm{~mm}$ \\
$R_{2}$ & $2.55 \mathrm{~mm}$ \\
$R_{3}$ & $5 / 3 \mathrm{~mm}$ \\
\hline Material of the stator: steel \\
\hline Density & $7900 \mathrm{~kg} / \mathrm{m}^{3}$ \\
Young's modulus & $2.1 \cdot 10^{11} \mathrm{~N} / \mathrm{m}^{2}$ \\
Poisson's ratio & 0.32 \\
\hline
\end{tabular}

where $M$ is the mass matrix, $C$ is the damping matrix, $K_{1}$ is the stiffness matrix, $K_{2}$ is the piezoelectric matrix, $K_{3}$ is the dielectric matrix, $F$ is the vector of nodal mechanical force, $Q$ is the vector of nodal electrical charge, $u$ is the nodal displacement vector, and $\Phi$ is the nodal potential vector.

Eq. (1) can be solved by applying FEM. Details are not presented here but easily can be found in the literature [4], [5]. Modeling was carried out using FEM software Ansys, which is used to create a solid finite element model and make different analyses. Simulations have been made in air, although other mediums could be considered while keeping the same methodology for the sensitivity analysis and the optimization. The next sections describe the operating principle as well as the modal shape and natural frequency obtained.

\section{B. Operating Principle and Characteristics of the Motor}

The FEM model of the motor is represented by Fig. 1 . Dimensions and materials used for the simulations are given in Table I and the Appendix. The operating principle of the motor is based on two perpendicular piezoelectric ceramics joined by a steel element (stator). These ceramics are activated by two electrical signals, which phase differs $90^{\circ}$ from each other. This generates on the top of the stator a flat elliptical motion (thanks to the standing wave cre-

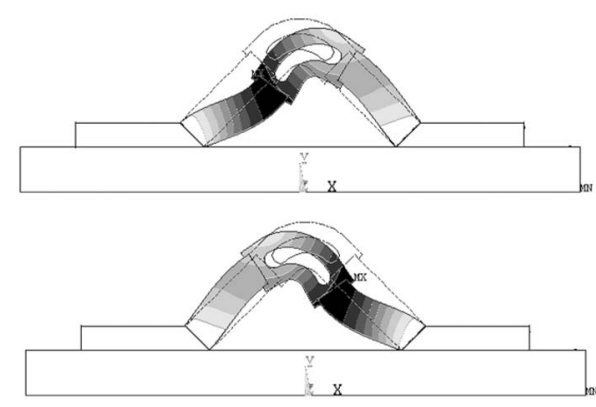

Fig. 2. Modal shape at resonance frequency.

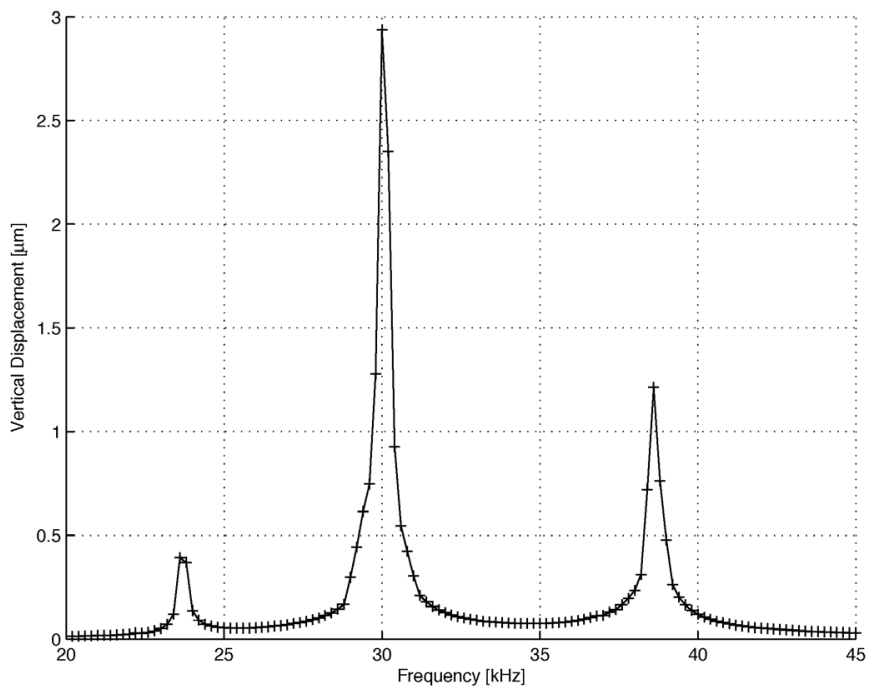

Fig. 3. Vertical displacement of a surface point.

ated). The application of a slider on the top of the stator produces a translation (linear motion).

The modal shape obtained and the vertical displacement as a function of the frequency are presented in Figs. 2 and 3 , respectively.

The harmonic response of the motor shows that the natural frequency is located at $30.09 \mathrm{kHz}$. With this frequency, elliptical displacement motion is obtained at the surface points of the motor as shown in Fig. 4.

Some experiments with a prototype built at the laboratory have been performed to confirm the numerical modeling and simulation. Materials used and dimensions of the motor are given in Table I and the Appendix. Fig. 5 shows the prototype.

The admittance (amplitude and phase) of the motor has been obtained using an impedance analyzer. This enables one to determine experimentally the value of the resonance frequency. Fig. 6 represents the measured admittance, and it shows that there are two resonance frequencies located at about $30 \mathrm{kHz}$ and $38 \mathrm{kHz}$, respectively. These values validate the results obtained by simulation (Fig. 3) and confirm that the resonance frequency that gives a maximum of deformation amplitude is indeed $30.09 \mathrm{kHz}$.

Moreover, the comparison of the vertical displacement (of a surface point of the motor) as a function of the ap- 


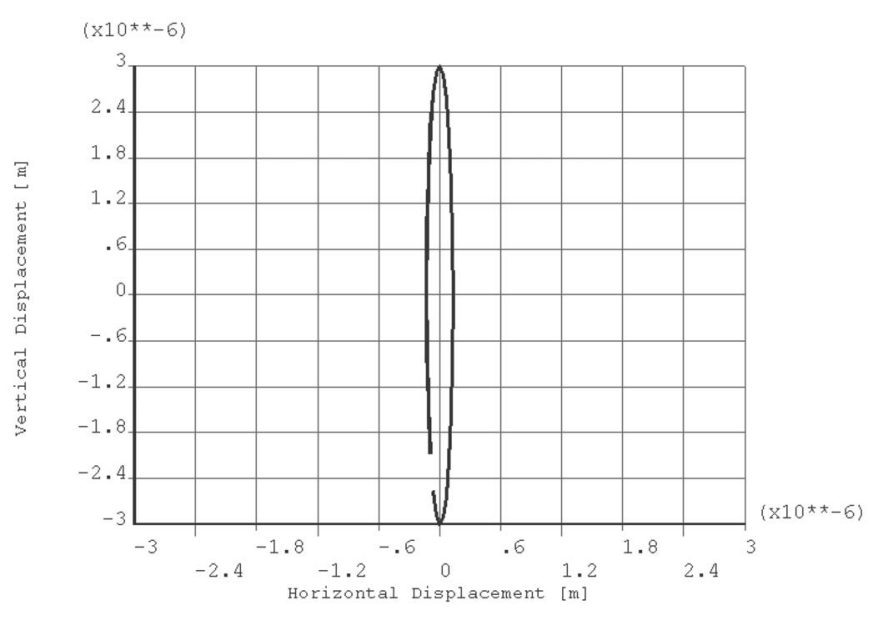

Fig. 4. Elliptic motion of a surface point.

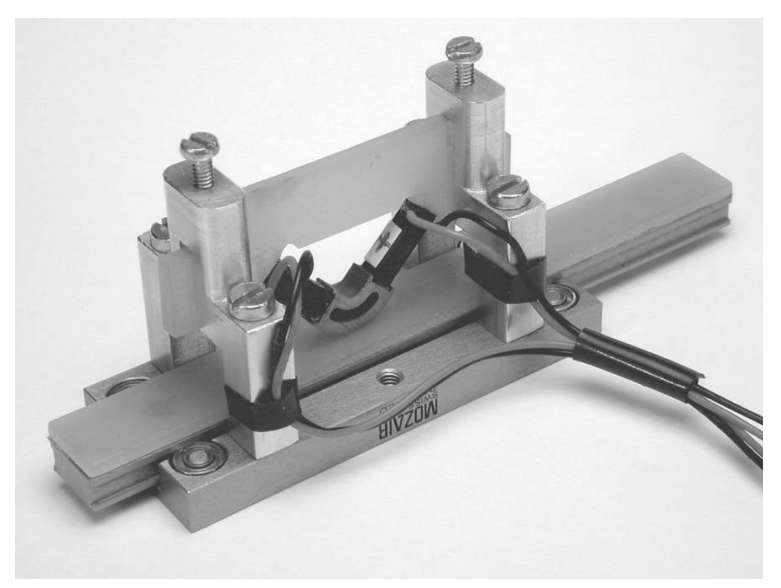

Fig. 5. Prototype of the motor realized in the laboratory.
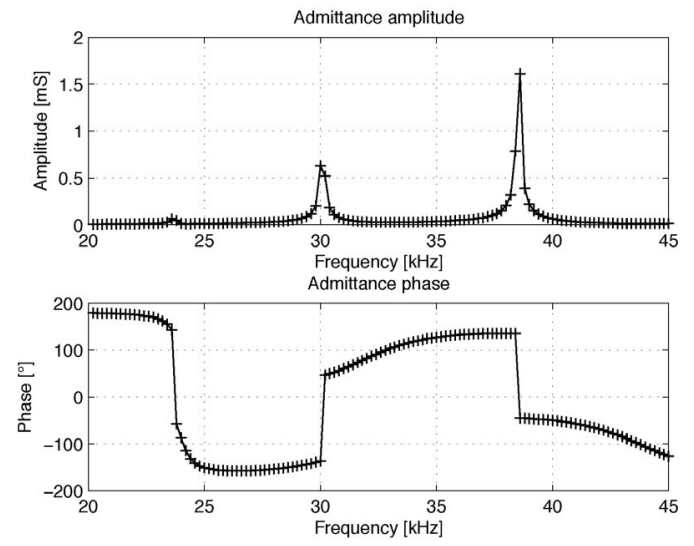

Fig. 6. Admittance versus frequency of the motor.

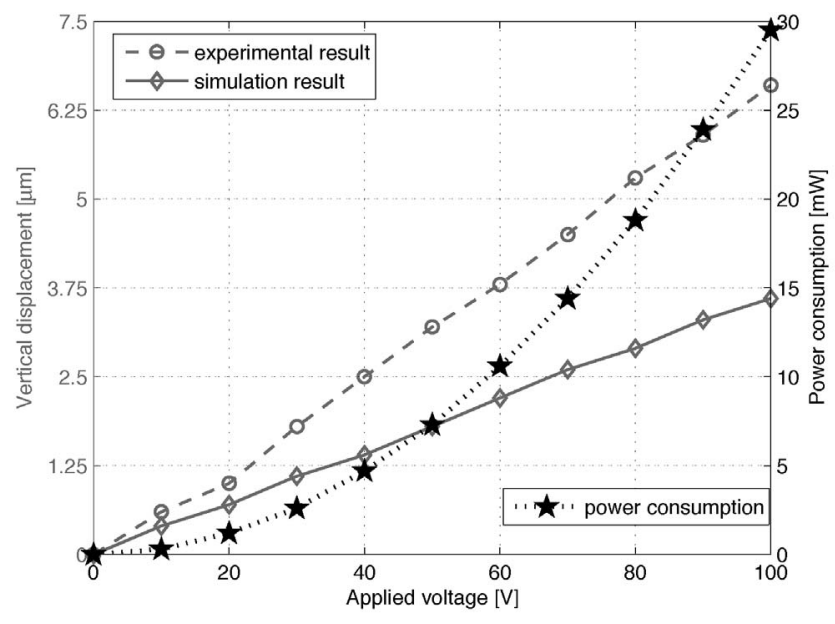

Fig. 7. Comparison of the vertical displacement versus applied voltage between Ansys simulation and experimental data and power consumption of the motor.

plied voltage at the resonance frequency between simulation and experiments has been carried out. The power consumption of the motor also has been measured. Results are presented in Fig. 7. They show that the model simulation results fit with the experimental values, although the attenuation factor used in the simulations should be determined precisely in order to be closer to reality.

As the results show, a deformation amplitude of about $6.6 \mu \mathrm{m}$ is obtained by applying a voltage of about $100 \mathrm{~V}$. The goal of the study is to reach a bigger deformation amplitude by changing the motor dimensions. This is the purpose of the next sections.

\section{Parametrization in Sight of Optimization}

For our study, the output parameter chosen for the optimization is the deformation amplitude (vertical displacement) of the vibrating piece (stator) describing the elliptical trajectory. The parametrization of the motor and the external parameters are described in Fig. 8, in which it is possible to obtain structures with or without a hole.

The different dimensional and external parameters of the motor are defined as follows:

- D: spacing between piezoceramics,

- B: width of piezoceramics,

- L: length of piezoceramics,

- H: thickness of piezoceramics,

- E: half-thickness of the stator,

- G: supplementary width added to the stator,

- $\alpha$ : angle defining stator's hole,

- $R_{1}$ : stator's inner radius,

- $R_{2}$ : hole's inner radius,

- $R_{3}$ : stator's outer radius,

- C: width of the hole,

- U: voltage applied to piezoceramics,

- F: frequency of the input voltage, 

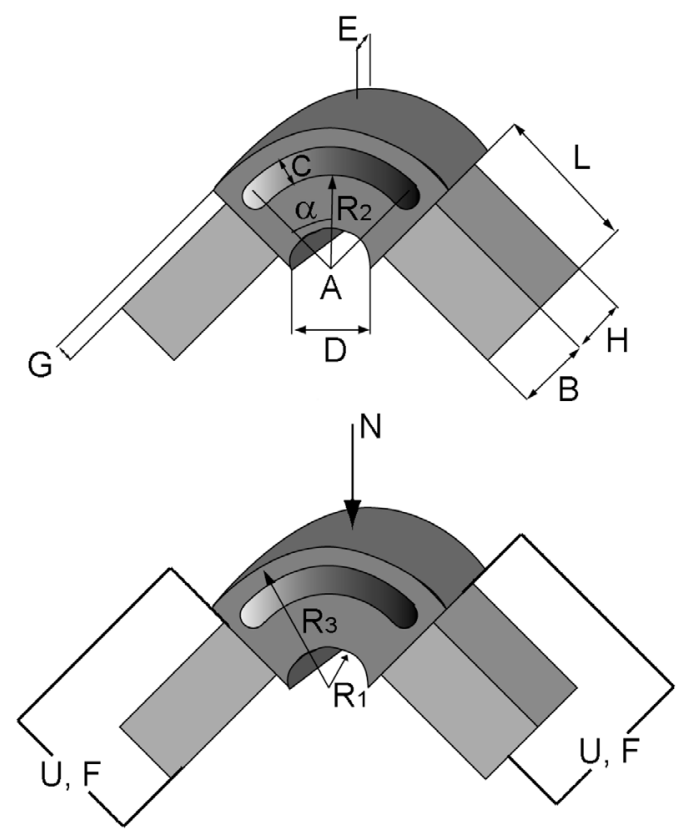

Fig. 8. Parametrization of the vibratory piece and external parameters.

TABLE II

Variation Ranges of the Different Input Parameters.

\begin{tabular}{lccc}
\hline Param. & Min $(-1)$ & Max $(+1)$ & Unit \\
\hline $\mathrm{U}\left(\equiv a_{1}\right)$ & 20 & 40 & {$[\mathrm{~V}]$} \\
$\mathrm{F}\left(\equiv a_{2}\right)$ & free & free & {$[\mathrm{Hz}]$} \\
$\mathrm{N}\left(\equiv a_{3}\right)$ & 0 & 50 & {$[\mathrm{~N}]$} \\
$\mathrm{D}\left(\equiv a_{4}\right)$ & 2 & 20 & {$[\mathrm{~mm}]$} \\
$\mathrm{B}\left(\equiv a_{5}\right)$ & 2 & 10 & {$[\mathrm{~mm}]$} \\
$\mathrm{H}\left(\equiv a_{6}\right)$ & 2 & 10 & {$[\mathrm{~mm}]$} \\
$\mathrm{L}\left(\equiv a_{7}\right)$ & 10 & 30 & {$[\mathrm{~mm}]$} \\
$\mathrm{E}\left(\equiv a_{8}\right)$ & 1 & 7 & {$[\mathrm{~mm}]$} \\
$\mathrm{G}\left(\equiv a_{0}\right)$ & 1 & 7 & {$[\mathrm{~mm}]$} \\
$\alpha\left(\equiv a_{10}\right)$ & 1 & 45 & {$\left[{ }^{\circ}\right]$} \\
$R_{1}{ }^{1}$ & 1 & 10 & {$[\mathrm{~mm}]$} \\
$R_{3}{ }^{2}$ & 4.8 & 31.8 & {$[\mathrm{~mm}]$} \\
$R_{2}\left(\equiv a_{11}\right)$ & $R_{1}+0.1 \cdot\left(R_{3}-R_{1}\right)$ & $R_{1}+0.6 \cdot\left(R_{3}-R_{1}\right)$ & {$[\mathrm{mm}]$} \\
$\mathrm{C}\left(\equiv a_{12}\right)$ & $0.1 \cdot\left(R_{3}-R_{1}\right)$ & $0.3 \cdot\left(R_{3}-R_{1}\right)$ & {$[\mathrm{mm}]$} \\
\hline
\end{tabular}

${ }^{1} R_{1}=\frac{D}{2}$.

${ }^{2} R_{3}=\sqrt{\left[\frac{\sqrt{2}}{2}(2 G+B)\right]^{2}+\left[\frac{\sqrt{2}}{2}(2 G+B)+\frac{D}{2}\right]^{2}}$

- N: prestressing force.

Once the dimensional parameters are chosen, it remains to define the variation range of each one. This must be done, of course, by respecting the physical constraints as well as the constraints imposed by the voltage $U$ applied to the piezoceramics and the prestressing force $N$. Table II gives the different variation ranges initially selected for the dimensional and external parameters to respect the constraints. These boundaries will be fitted more precisely thanks to the stage of preoptimization presented in the following section.

\section{PREoptimization}

This stage of the study consists in analyzing the influence of each input parameter on the deformation amplitude of the vibrating piece of the motor. To do this, design of experiments (DOE) is used. This technique makes it possible to characterize the influences of the parameters taken individually (order 1) but also those coupled with each other (order 2, 3, etc.). In this manner, it is possible to determine which parameters will be kept as variables (and so define a more precise variation range) and which will be fixed during the optimization stage.

\section{A. Sensitivity Analysis}

Through the use of more or less complex algorithms, the sensitivity analysis using design of experiments allows one to reduce the number of simulations while obtaining a maximum of information. There are several kinds of experimental designs [6], [7]. Only two types will be used in this study: factorial designs and the Doehlert method. The sensitivity analysis is performed according to the following steps:

- choice of the parameters,

- random draw or according to an experimental design of $N$ sets of values of the parameters,

- simulations,

- storage of the output results to be analyzed,

- determination of the most dominating parameters.

These different steps are carried out by using two programs: Matlab and Ansys ${ }^{1}$. The first allows one to create simulation files by varying the input parameters according to the selected method. Once these files are created, they are used in a reference file that is launched $N$ times toward the finite element program Ansys via Matlab in batch mode. At the end of each simulation, a result file is created by Ansys and treated with Matlab, which stores the results obtained in a vector. This answer vector then is analyzed to determine the most dominating input parameters. Fig. 9 describes the routine used as well as the various interactions between the two softwares.

\section{B. Sensitivity Analysis Methods}

1. Factorial Design: This method consists of choosing the simulation points at the edge of the multidimensional domain defined by the input parameter ranges and fitting the simulation results to an appropriate polynomial function corresponding to a Taylor series of the model being analyzed [8]. Full factorial design allows one to determine all coefficients of a linear model with all possible interactions within $2^{N}$ runs (2). This design methodology has the disadvantage of requiring a lot of runs and is practicable

\footnotetext{
${ }^{1}$ Ansys Multiphysics 8.1 version, User manual, Ansys Inc., Canonsburg, PA, 2004.
} 

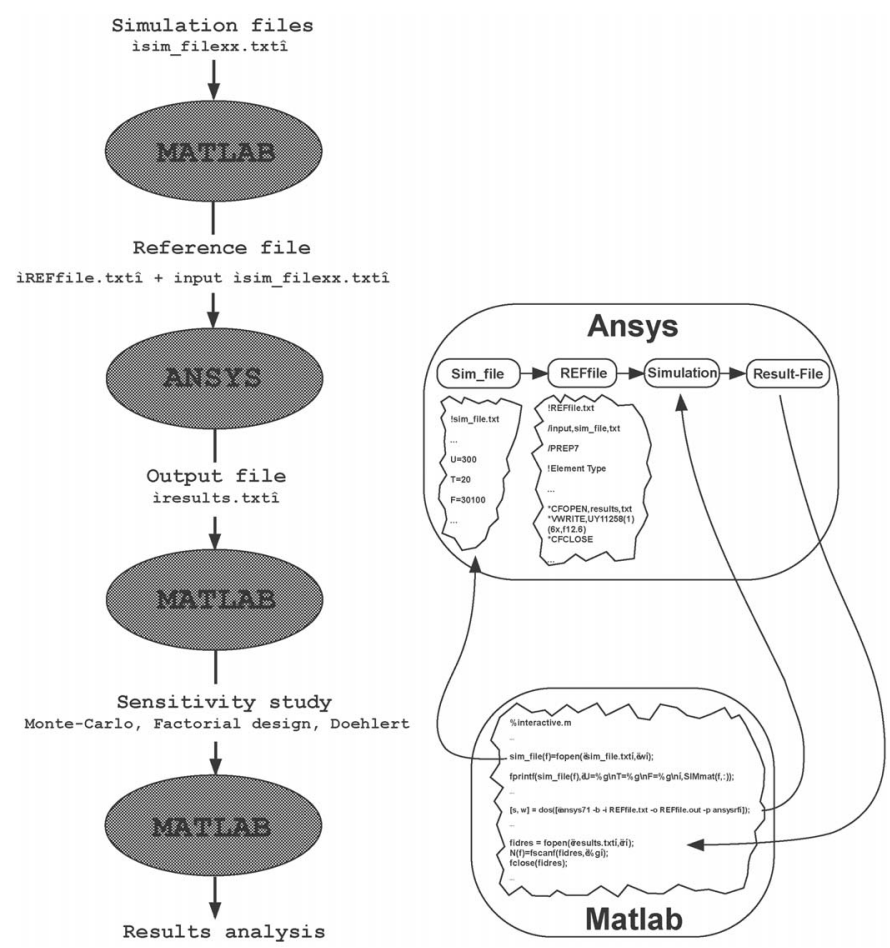

Fig. 9. Steps of the sensitivity analysis.

only for a small number of input parameters:

$$
\begin{aligned}
Y(x) & =a_{0}+\sum_{i=1}^{N} a_{i} x_{i}+\sum_{i \neq j}^{N} a_{i j} x_{i} x_{j}+\cdots \\
& \cdots+\sum_{i \neq j \neq k}^{N} a_{i j k} x_{i} x_{j} x_{k}+\cdots+a_{i \ldots N} x_{i} \ldots x_{N} .
\end{aligned}
$$

The coefficients $a_{0}, a_{1}, \ldots$ are called the effects of the $x_{i}$ factors. One makes the distinction between:

- $a_{0}$ : constant effect (equal to the experiments mean),

- $a_{i}$ : principal effects,

- $a_{i j}$ : effects of the first order interactions,

- $a_{i j k}$ : effects of the second order interactions.

The linear meta-model (whose coefficients are $\left[a_{0}, a_{1}, a_{2} \ldots\right]=C$ ) is fitted on the results (defined in a matrix $R$ ) using a model matrix $X$ by the operations given by (3):

$$
X C=R \Rightarrow C=\left(X^{T} X\right)^{-1} X^{T} R .
$$

2. Doehlert Design (Response Surfaces): The second selected method, usually called Doehlert network, makes it possible to easily move the center of interest in an experimental space (through an iterative way) according to the results ([9], [10]). Moreover, this design method is very interesting for optimization problems. As an example, the two dimensional Doehlert's network (two input parameters) and its corresponding model matrix $X$ are represented in Fig. 10.

The calculation methodology used in the factorial method also can be applied to Doehlert design (only the
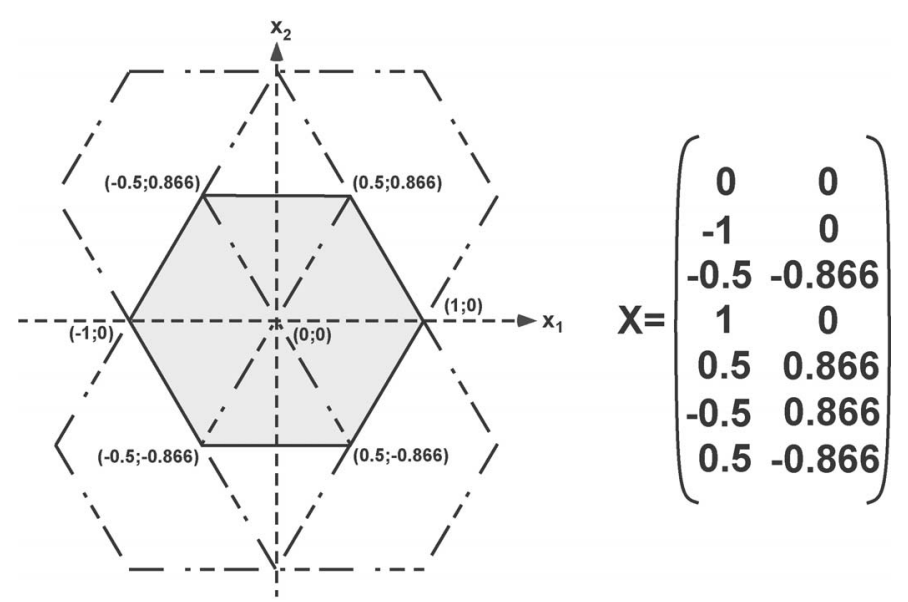

Fig. 10. Two-dimensional Doehlert's network and the corresponding model matrix.

model matrix is different). The larger the number of input parameters, the larger the number of simulations $N$ is, and thus the experimental matrix is bigger. For a more precise analysis of our system, it would be judicious to consider more simulation points besides lower and upper bounds of the variables variation ranges, but the simulation time then would be larger. This is why, in a first approximation, less simulation points have been taken into account.

In the next section, the results obtained applying these two methods are presented. The different input parameters selected vary according to the case in which one is structure with or without a hole. The output parameter is the deformation amplitude obtained at the top of a stator surface point (vertical displacement). The analysis of the results will be done using two types of graphs. First, the graphs representing the effects. It is a graphical representation of the coefficients $a_{i}, a_{i j}, a_{i j k}, \ldots$ shown in (2). This representation just makes it possible to see the parameters having the most important influence on the vertical displacement of the stator. As the number of analyzed parameters is large, only those being the most influential will be indicated by arrows on the graphs. It should be noted that, in this case, the analysis of the effects will be limited to those going until order 2 . The quadratic effects also will be taken into account. The others (order 4 and higher) will be neglected.

\section{Results}

First of all, an analysis of the input parameters effects on the deformation amplitude has been carried out. The results obtained are represented in Figs. 11, 12, and 13 for a structure without a hole.

The graph representing the influences is based on the results obtained and given by the graphs of the effects. To better understand and see which are the most influential parameters, a program (Matlab) has been implemented, and the influence of each parameter is calculated then normalized in relation to the most influential one. In this manner, a more representative graph showing the pa- 
Principal relative effects

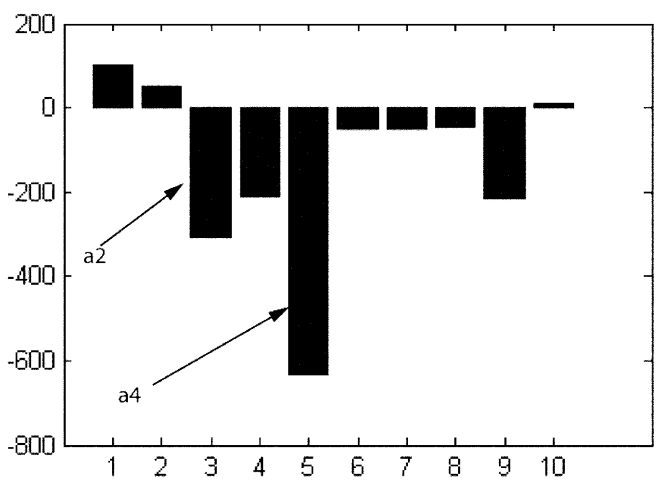

Fig. 11. Relative effects of the different input parameters for a structure without a hole; principal effects.

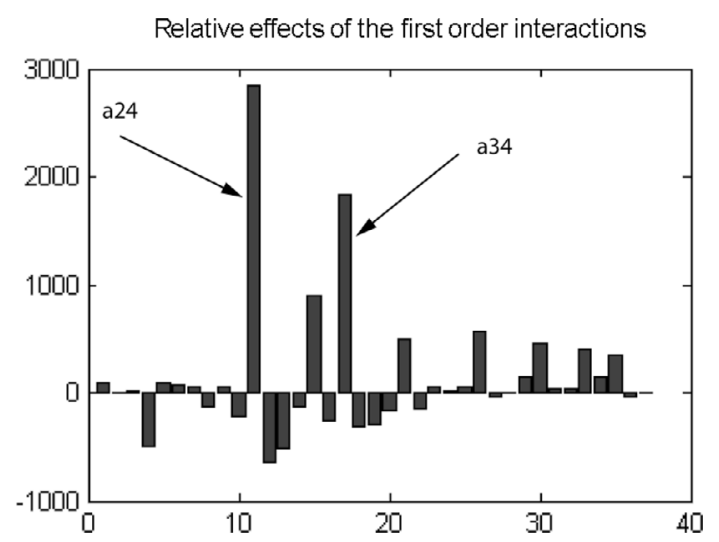

Fig. 12. Relative effects of the different input parameters for a structure without a hole; first order interactions effects.

rameters that are the most important is obtained. This is shown in Fig. 14.

The same methodology has been applied for a structure with a hole. The results are presented in Figs. 15, 16, and 17 .

\section{Comments}

The results obtained for both structures, with and without a hole, are very interesting. It can be noticed that the influence of this hole is very important, as well as from a point of view of the deformation amplitude as of the various influences of the input parameters. Indeed, in the case of a structure without a hole, the most significant input parameter is the voltage $U$ applied to the piezoceramics. One can easily understand this result because of the direct relation between the displacement of the stator, the dilation of the piezoceramics, and the applied voltage. Indeed, it is obvious that the larger the applied voltage the bigger the dilation and the displacement. Furthermore, the halfthickness of the stator $E$ also has an important effect on the deformation amplitude, and the other input parameters have a more or less equal influence but definitely lower than that of $U$ and $E$. However, for a structure with a hole,
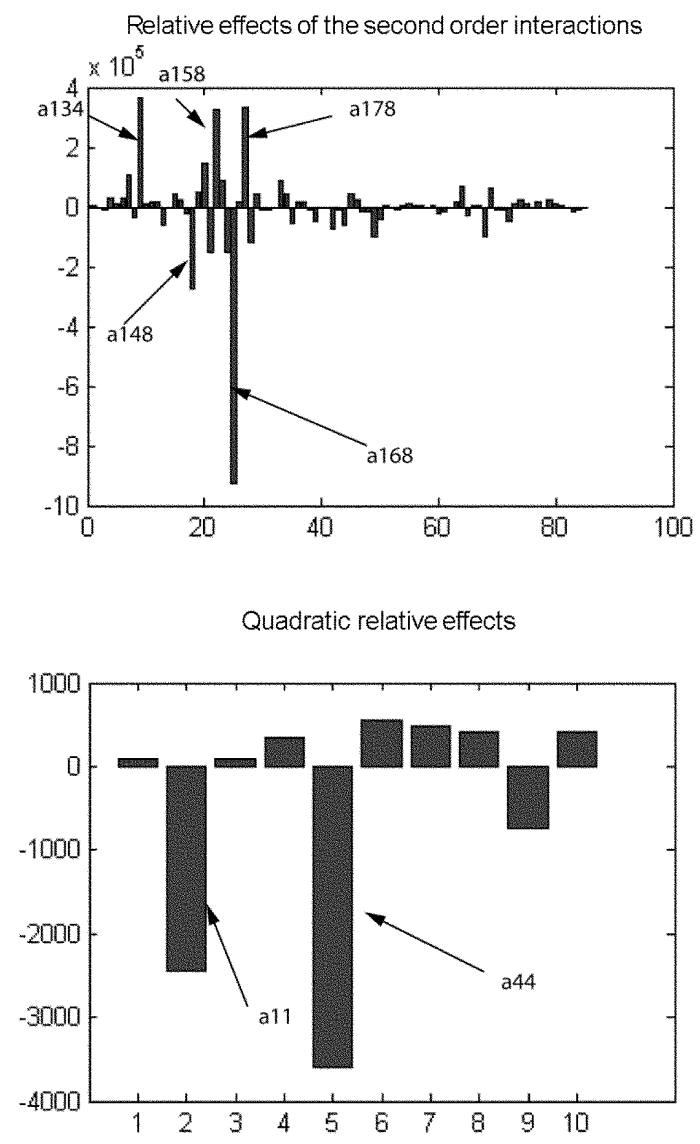

Fig. 13. Relative effects of the different input parameters for a structure without a hole: (top) second order interactions effects, (bottom) quadratic effects.

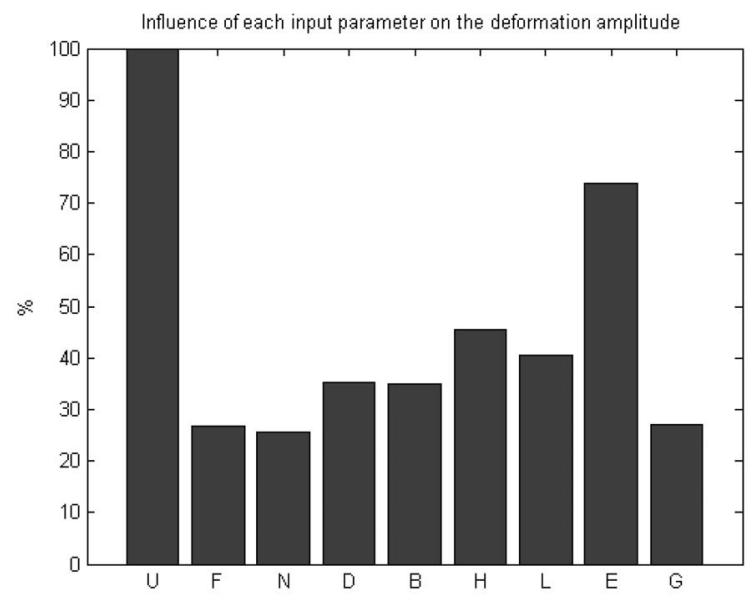

Fig. 14. Input parameters influences for a structure without a hole. 
a)

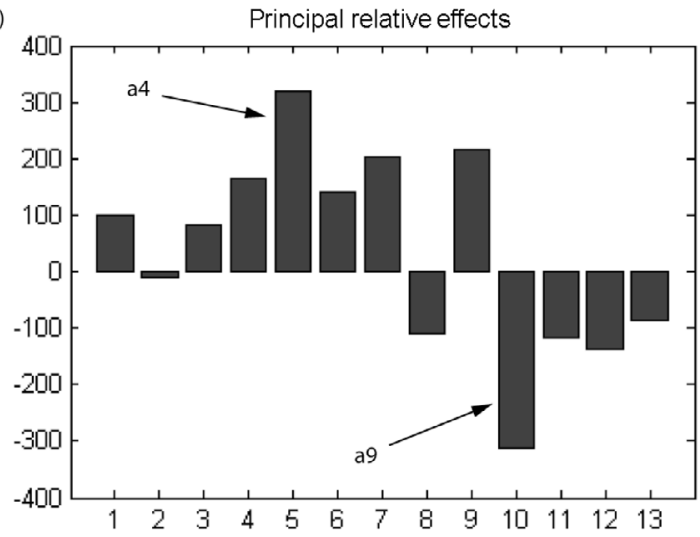

b)

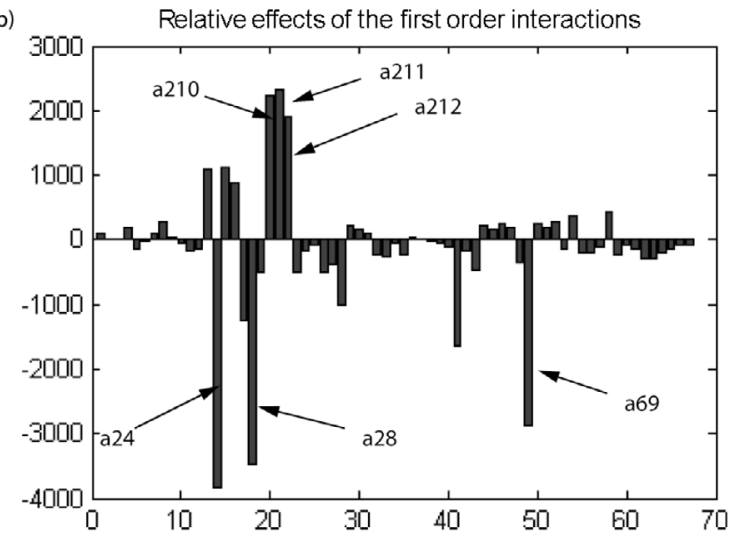

Fig. 15. Relative effects of the different input parameters for a structure with a hole: (a) principal effects, (b) first order interactions effects.

the frequency $F$ has a more influential effect than the applied voltage (see Fig. 17). This can be explained by the fact that the addition of a hole generates a considerable modification of the stator structure, and thus its resonance frequency. But, in the case of a structure without a hole, the stator remains overall identical, only the variation of its thickness can lead to a slight modification of the resonance frequency, but not as important as in the case of the presence of a hole.

Moreover, the displacement amplitudes obtained in both cases are quite different. Indeed, the values for a structure with a hole are much more often positive than those obtained for a structure without a hole, but also bigger $(20 \mu \mathrm{m}$ compared to $10 \mu \mathrm{m})$. Table III, Figs. 18 and 19 summarize the different results obtained.

It should be noted that there is a clear difference of calculation condition between a structure with a hole and one without. Indeed, the simulation of a structure like that presented in Fig. 18 takes three times less computing time than the simulation of a structure with a hole (Fig. 19). The size of the mesh also is smaller in the area of the hole, which implies an increase in the computing time. Moreover, there are more parameters in a structure with a hole, which increases the computing time to a value of approximately 8 minutes per simulation.

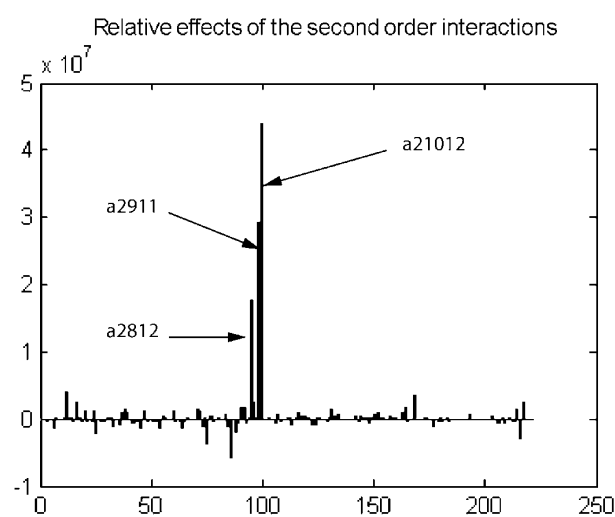

Quadratic relative effects

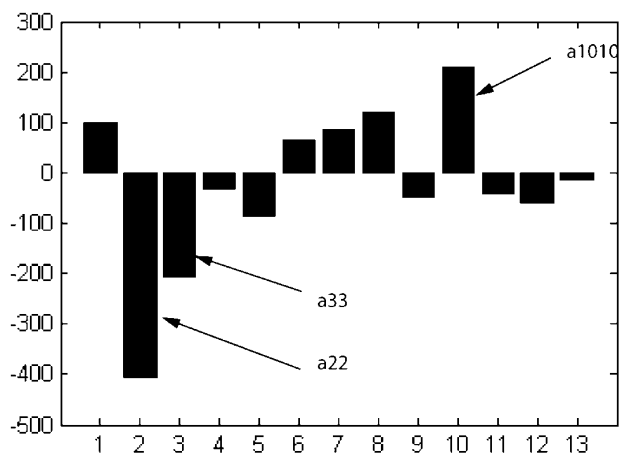

Fig. 16. Relative effects of the different input parameters for a structure with a hole: (top) second order interactions effects, (bottom) quadratic effects.

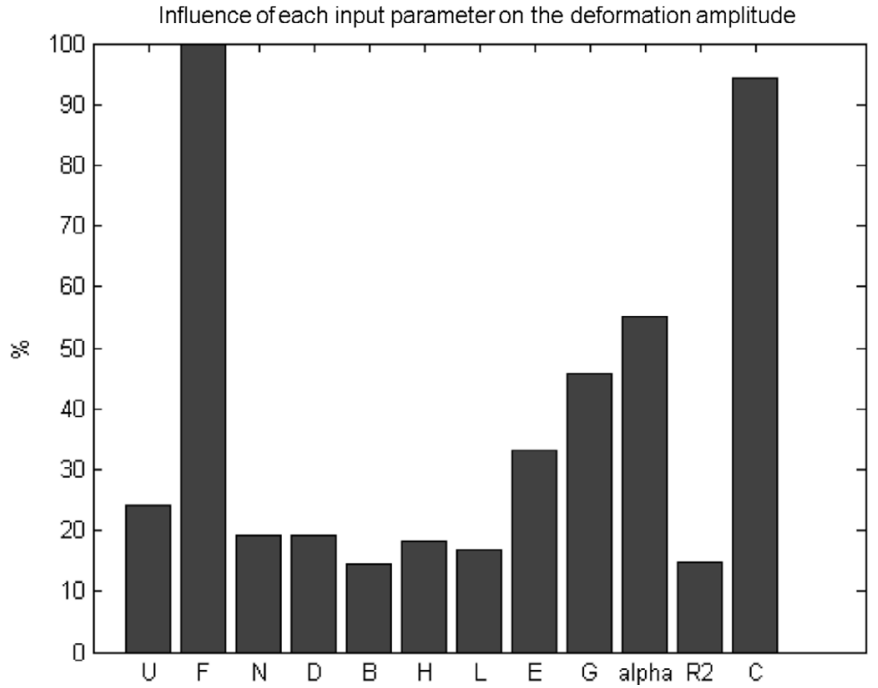

Fig. 17. Input parameters influences for a structure with a hole. 
TABLE III

Input Parameters and Maximum Deformation Amplitude.

\begin{tabular}{lll}
\hline & Without a hole & With a hole \\
Max. amplitude & $9.1 \mu \mathrm{m}$ & $22.6 \mu \mathrm{m}$ \\
\hline External parameters & $U=30 \mathrm{~V}$ & $U=30 \mathrm{~V}$ \\
& $F=7.38 \mathrm{kHz}$ & $F=12.0 \mathrm{kHz}$ \\
& $N=60.2 \mathrm{~N}$ & $N=50 \mathrm{~N}$ \\
Dimensional parameters & $D=18.1 \mathrm{~mm}$ & $D=11 \mathrm{~mm}$ \\
& $B=6 \mathrm{~mm}$ & $B=6 \mathrm{~mm}$ \\
& $H=6 \mathrm{~mm}$ & $H=8.6 \mathrm{~mm}$ \\
& $L=20 \mathrm{~mm}$ & $L=19.1 \mathrm{~mm}$ \\
& $E=4 \mathrm{~mm}$ & $E=3.7 \mathrm{~mm}$ \\
& $G=4 \mathrm{~mm}$ & $G=1.8 \mathrm{~mm}$ \\
& & $\alpha=23^{\circ}$ \\
& & $R_{2}=8.5 \mathrm{~mm}$ \\
& $C=1.7 \mathrm{~mm}$ \\
\hline
\end{tabular}
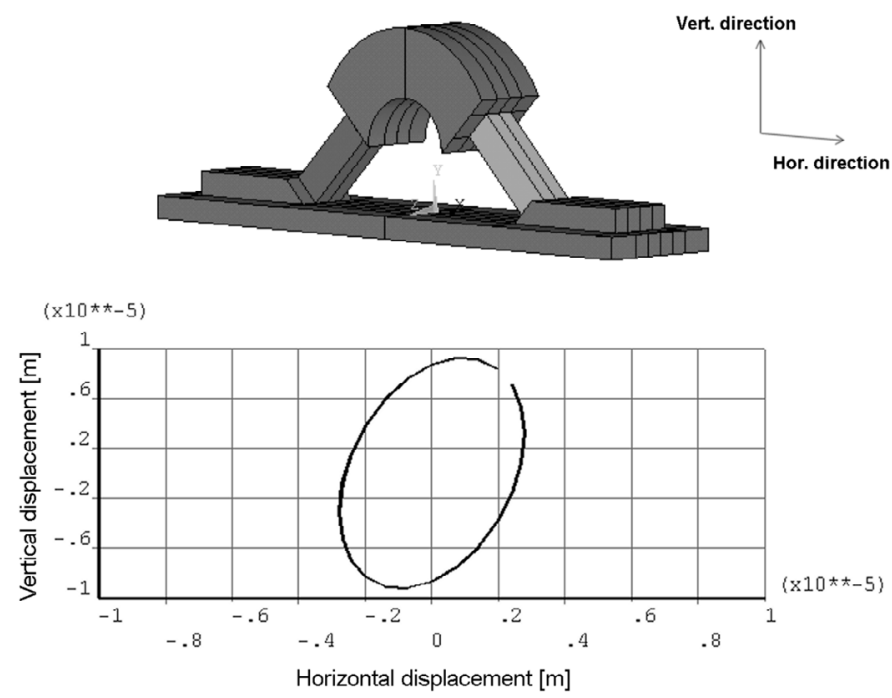

Deformation amplitude: $9.1 \mu \mathrm{m}$

Fig. 18. Structures comparison: maximum amplitude without a hole.

The results found in the preoptimization stage then can be applied to the FE optimization, which is described in the following section.

\section{FE OptimizATION}

\section{A. Introduction}

The process involved in a FE optimization consists of following precise steps. An analysis file to be used during looping first must be created. It will build the model parametrically, obtain the solution, and retrieve and assign to parameters the response quantities that will be used as state variables and objective function. The next step is to declare the optimization variables (+ objective function) and choose an optimization method. Two different approaches have been chosen in our case: the first order method and the factorial design tool.
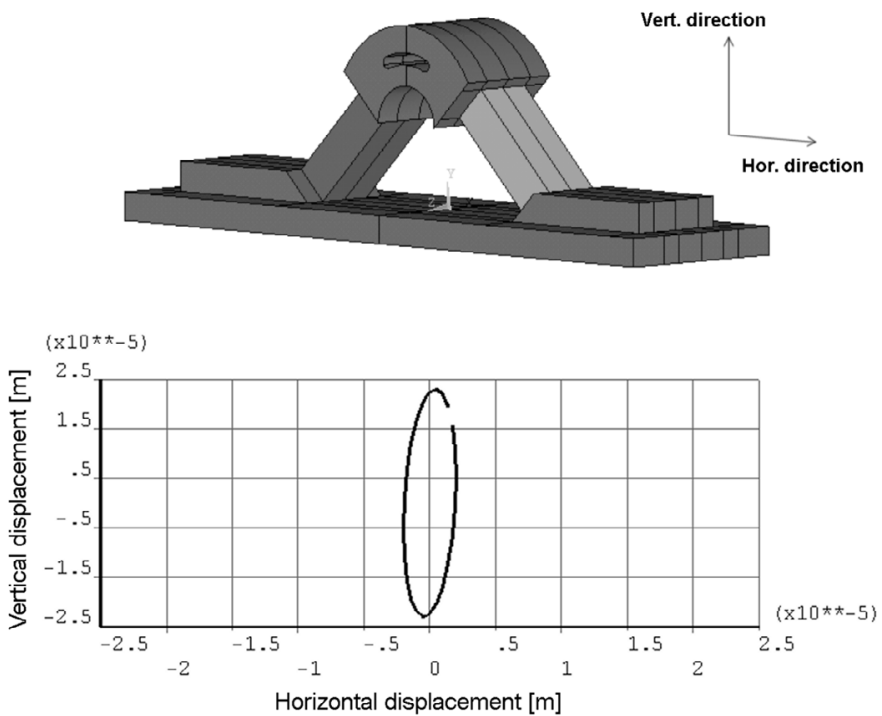

Deformation amplitude: $22.6 \mu \mathrm{m}$

Fig. 19. Structures comparison: maximum amplitude with a hole.

\section{B. First Order Method}

First order methods use gradients of the dependent variables with respect to the design specifications. The Gauss-Newton method is a class of methods for solving this type of problem. An improved and efficient version of the method is the so-called Levenberg-Marquardt algorithm. For a detailed discussion of these methods, see [11].

\section{Factorial Tool}

Factorial design tool uses a two-level, full or fractional factorial evaluation to generate design set results at all extreme points of the design space ${ }^{1}$ [7]. In our case, a full evaluation will be performed: the program realizing $2 n$ loops, where $n$ is the number of design variables. Every component of the design variable vector will take two extreme values (maximum and minimum). So, in a full factorial evaluation, every combination of design variable extreme values is considered in the $n$-dimensional design space.

In the next section, the results obtained using both methods are presented and compared.

\section{Results}

The structure chosen for the FE optimization is represented in Fig. 18. The choice to simulate a structure without a hole is made because the computing time is definitely shorter as there are fewer parameters, and thus fewer iterations to perform. The maximum amplitude obtained in the preoptimization stage using the Doehlert design method is $9.1 \mu \mathrm{m}$. The external and dimensional parameters given in Table III have been taken as initial variables, and variation ranges have been chosen as shown in Table IV. The objective function is obviously the deformation amplitude. 
TABLE IV

Initial Values and VARiation RANGes of the InPut PARAMEters FOR the FE Optimization.

\begin{tabular}{ccc}
\hline Parameter & Initial value & Variation range \\
\hline $\mathrm{U}$ & $30 \mathrm{~V}$ & fixed \\
$\mathrm{F}$ & $7.38 \mathrm{kHz}$ & {$[5.9 \mathrm{kHz} ; 8.9 \mathrm{kHz}]$} \\
$\mathrm{N}$ & $60.2 \mathrm{~N}$ & fixed \\
$\mathrm{D}$ & $18.1 \mathrm{~mm}$ & {$[18.0 \mathrm{~mm} ; 18.2 \mathrm{~mm}]$} \\
$\mathrm{B}$ & $6 \mathrm{~mm}$ & {$[5 \mathrm{~mm} ; 7 \mathrm{~mm}]$} \\
$\mathrm{H}$ & $6 \mathrm{~mm}$ & {$[5 \mathrm{~mm} ; 7 \mathrm{~mm}]$} \\
$\mathrm{L}$ & $20 \mathrm{~mm}$ & {$[15 \mathrm{~mm} ; 25 \mathrm{~mm}]$} \\
$\mathrm{E}$ & $4 \mathrm{~mm}$ & {$[3.9 \mathrm{~mm} ; 4.1 \mathrm{~mm}]$} \\
$\mathrm{G}$ & $4 \mathrm{~mm}$ & Tol.: $0.01 \mathrm{~mm}$ \\
\hline \multicolumn{3}{c}{}
\end{tabular}

The first order and fractional methods have been applied. Figs. 20 and 21 show the results obtained with both methods.

\section{E. Discussion}

As the results show, the preoptimization stage already allows an improvement of the deformation amplitude of the motor-vibrating body, but FE optimization makes it possible to obtain a bigger deformation amplitude. Results obtained with the first-order method give a bigger deformation amplitude, which shows that this optimization method is better than the factorial one, although computing time is much longer. The structure optimized with Ansys does not have a hole, and within sight of the results obtained in the preoptimization stage, the FE optimization of a structure with a hole will give even better results, although the computing time necessary for the analysis of a single case study is very long and does not yet converge. Moreover, the structures presented in this paper have resonance frequencies that are not in the ultrasonic range; this could be obtained by restricting the variation range of the frequency $F$ to frequencies higher than $20 \mathrm{kHz}$. Nevertheless, methodology used appears to be very effective and already allows one to multiply the deformation amplitude by a factor higher than 10 (from $3.3 \mu \mathrm{m}$ to $33.6 \mu \mathrm{m}$ ) compared to the initial design model, and reduce the voltage applied to the piezoceramics by a factor of 3 (from $100 \mathrm{~V}$ to $30 \mathrm{~V})$.

\section{Conclusions}

Simulation works should incorporate a sensitivity analysis indicating the confidence limits of output data. This can be done efficiently by using up-to-date statistical techniques as it is done in other fields [12]. Sensitivity analysis also must be considered when developing models as well as when beginning a new study. In our case, some of these techniques have been applied to carry out the sensitivity analysis of a piezoelectric motor.

Design of experiments applied as a preoptimization
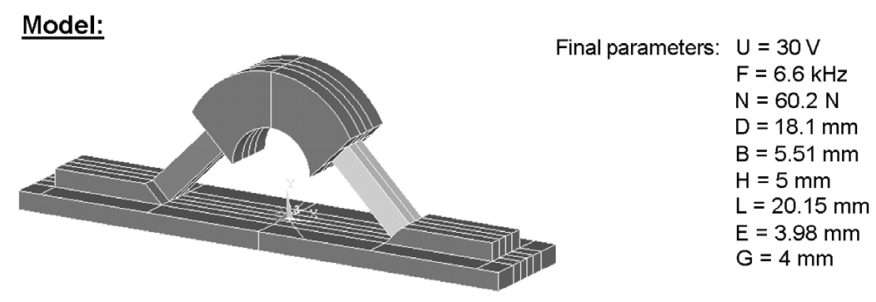

Deformation:
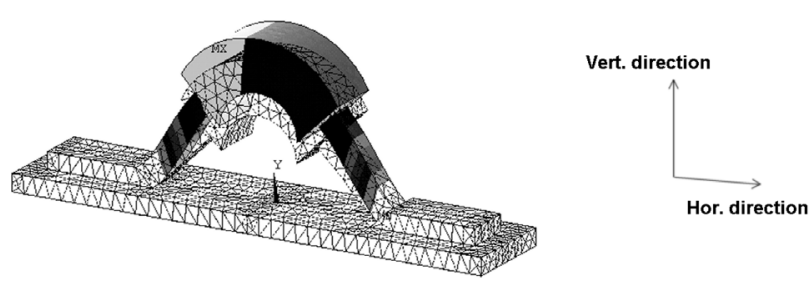

Elliptical trajectory of a surface point:

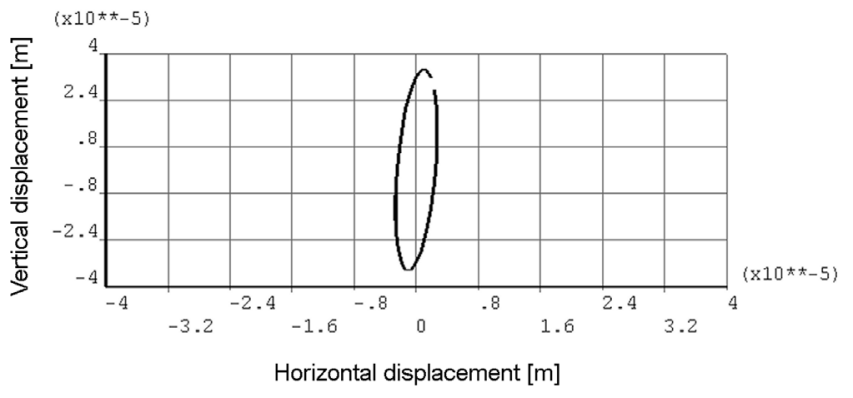

Deformation amplitude: $33.6 \mu m$

Fig. 20. FE optimization; first order method.

stage and then FE optimization method are a very interesting way to maximize the deformation amplitude of an ultrasonic motor and show good complementarity. Design of experiments makes it possible to simplify the problem by reducing the variation ranges of the input parameters used to carry out the FE optimization. Nevertheless, a feasibility study should be achieved before designing the motor. Indeed, some structures found by applying our methodology should be the subject of a thorough analysis by means of the mechanical constraints acting on the vibrating piece, in particular by realizing various prototypes for experimental measurements. However, design methodology and optimization methods described in this paper allow one to explore a new way in the optimization field of smart ultrasonic actuators [13].

\section{Appendix A}

Material data of the used stacked PZT ceramic NLA$2 \times 3 \times 9$ from Tokin Company, Tokyo, Japan.

Density:

$$
\rho=7700 \mathrm{~kg} / \mathrm{m}^{3} .
$$


Model:

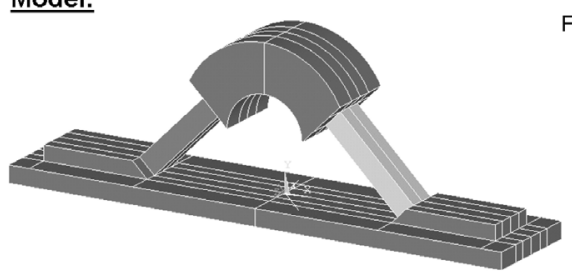

Final parameters: $U=30 \mathrm{~V}$ $\mathrm{F}=5.9 \mathrm{kHz}$ $\mathrm{N}=60.2 \mathrm{~N}$ $\mathrm{D}=18.1 \mathrm{~mm}$ $B=5 \mathrm{~mm}$ $\mathrm{H}=7 \mathrm{~mm}$ $\mathrm{L}=25 \mathrm{~mm}$ $E=5 \mathrm{~mm}$ $\mathrm{G}=4 \mathrm{~mm}$

\section{Deformation:}

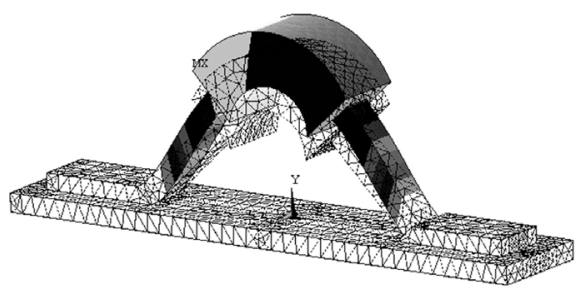

Vert. direction

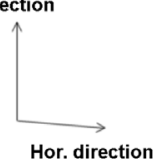

Elliptical trajectory of a surface point:

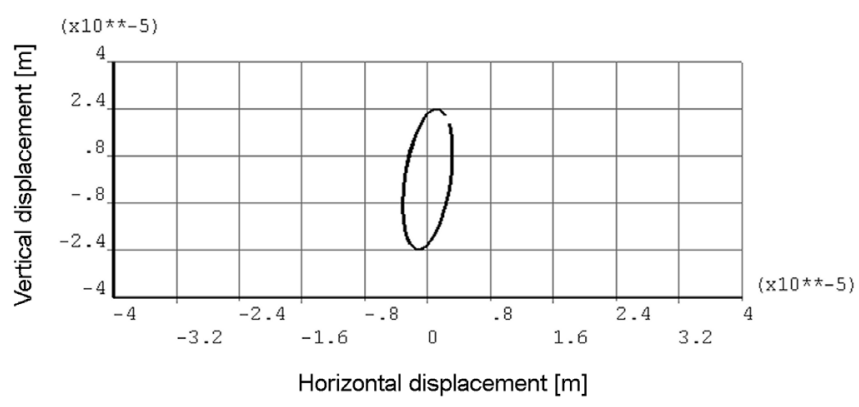

Deformation amplitude: $24.1 \mu \mathrm{m}$

Fig. 21. FE optimization; factorial method.

Stiffness matrix:

$$
c^{E}=\left[\begin{array}{cccccc}
12.8 & 6.8 & 6.6 & 0 & 0 & 0 \\
& 12.8 & 6.6 & 0 & 0 & 0 \\
& & 10.0 & 0 & 0 & 0 \\
& & & 2.1 & 0 & 0 \\
& & & & 2.1 & 0 \\
& & & & & 2.1
\end{array}\right] \cdot 10^{10} \mathrm{~N} / \mathrm{m}^{2} .
$$

Piezoelectric constants:

$$
e=\left[\begin{array}{cccccc}
0 & 0 & 0 & 0 & 11.8 & 0 \\
0 & 0 & 0 & 11.8 & 0 & 0 \\
-6.1 & -6.1 & 15.7 & 0 & 0 & 0
\end{array}\right] \quad \mathrm{As} / \mathrm{m}^{2} .
$$

Dielectric constants:

$$
\epsilon^{S}=\left[\begin{array}{ccc}
8.8 & 0 & 0 \\
0 & 8.8 & 0 \\
0 & 0 & 8.8
\end{array}\right] \cdot 10^{-9} \mathrm{As} / \mathrm{Vm} .
$$

\section{REFERENCES}

[1] T. Sashida and T. Kenjo, An Introduction to Ultrasonic Motors. Oxford: Clarendon Press, 1993.

[2] S. Ueha and Y. Tomikawa, Ultrasonic Motors - Theory and Applications. Oxford: Clarendon Press, 1993.

[3] J.-M. Fuerbringer and A. Persily, "Sensitivity Analysis of VOC Measurement," Lausanne, Switzerland: Ecole Polytechnique Fédérale de Lausanne (EPFL), 2004.

[4] N. N. Abboud, G. L. Wojcik, D. K. Vaughan, J. Mould, D. J. Powell, and L. Nikodym, "Finite element modelling for ultrasonic transducers," in Proc. Int. Symp. Med. Imaging, SPIE, 1998, pp. 19-42.

[5] R. Lerch, "Simulation of piezoelectric devices by two-and threedimensional finite elements," IEEE Trans. Ultrason., Ferroelect., Freq. Contr., vol. 37, pp. 233-247, 1990.

[6] G. E. P. Box, W. G. Hunter, and J. S. Hunter, Statistics for Experimenters, An Introduction to Design, Data Analysis and Model Building. New York: Wiley, 1978.

[7] D. C. Montgomery, Design and Analysis of Experiments. New York: Wiley, 1997.

[8] B. H. Gunter, "How experimental design concepts can improve experimentation in the physical sciences," Comput. Phys., vol. 7, no. 3, pp. 262-272, May/June 1993.

[9] G. E. P. Box and N. Draper, Empirical Model Building and Response Surfaces, Wiley Series in Probability and Mathematical Statistics. New York: Wiley, 1987.

[10] D. Feneuille, D. Mathieu, and R. Phan-Tan-Luu, "Méthodologie de la recherche expérimentale," Aix-en-Provence, France: Laboratoire de Prospective Réactionnelle et d'Analyse de l'Information, 1983. (in French)

[11] J. E. Dennis and R. B. Schnabel, Numerical Methods of Unconstrained Optimization and Nonlinear Equations. Englewood Cliffs, NJ: Prentice-Hall, 1983.

[12] J.-M. Fuerbringer and C.-A. Roulet, "Comparison and combination of factorial and Monte-Carlo design in sensitivity analysis," Building Environment, vol. 30, no. 4, pp. 505-519, 1995.

[13] M. I. Frecker, "Recent advances in optimization of smart structures and actuators," J. Int. Mater. Syst. Struct., vol. 14, pp. 207-216, Apr./May 2003.

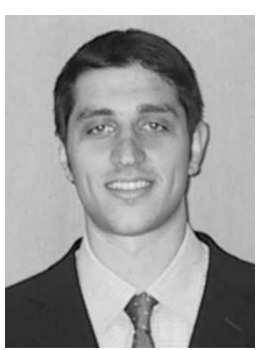

José M. Fernandez was born in Lausanne in 1977. He received the M.Sc. degree in electricity engineering from the Swiss Federal Institute of Technology - Lausanne (EPFL) in 2002. He is currently working at the Laboratory of Integrated Actuators as a research assistant and Ph.D. student.

His research activities are mainly the study and comparison of various linear ultrasonic motors, using either the standing wave or the traveling wave method. He is also supporting ANSYS software for EPFL users as well as co-organizing related courses within the school.

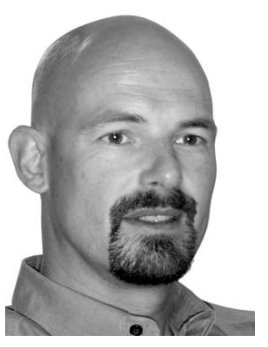

Yves Perriard was born in Lausanne in 1965. He received the M.Sc. degree in microengineering in 1989 and the Ph.D. degree in 1992, both from the Swiss Federal Institute of Technology-Lausanne (EPFL).

Cofounder of Micro-Beam SA, he was $\mathrm{CEO}$ of this company involved in high precision electric drive. Senior lecturer from 1998 and professor since 2003, he is currently director of the Integrated Actuator Laboratory at EPFL. His research interests are in the field of new actuator design and associated electronic devices. He is author and co-author of more than 40 publications and patents. 